\title{
A Systematic Review and Meta-Analysis of Nature-Based Mindfulness: Effects of Moving Mindfulness Training into an Outdoor Natural Setting
}

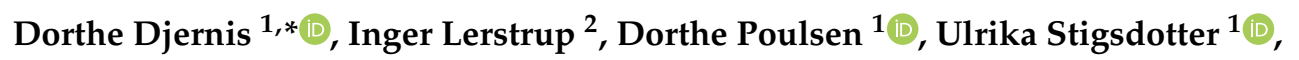 \\ Jesper Dahlgaard ${ }^{3,4}$ and Mia O'Toole ${ }^{5}$ \\ 1 Department of Geoscience and Natural Resource Management, University of Copenhagen, \\ C 1958 Frederiksberg, Denmark \\ 2 Department of Landscape Architecture and Management, Swedish University of Agricultural Sciences, \\ 23053 Alnarp, Sweden \\ 3 Department of Clinical Medicine, Aarhus University, N 8200 Aarhus, Denmark \\ 4 Research Center for Health and Welfare Technology, VIA University College, N 8200 Aarhus, Denmark \\ 5 Department of Psychology and Behavioural Sciences, Aarhus University, C 8000 Aarhus, Denmark \\ * Correspondence: ddj@ign.ku.dk; Tel.: +45-6168-8011
}

Received: 7 August 2019; Accepted: 28 August 2019; Published: 2 September 2019

check for updates

\begin{abstract}
Research has proven that both mindfulness training and exposure to nature have positive health effects. The purpose of this study was to systematically review quantitative studies of mindfulness interventions conducted in nature (nature-based mindfulness), and to analyze the effects through meta-analyses. Electronic searches revealed a total of 25 studies to be included, examining 2990 participants. Three analyses were conducted: Nature-based mindfulness interventions evaluated as open trials $(k=13)$, nature-based mindfulness compared with groups in non-active control conditions $(\mathrm{k}=5)$, and nature-based mindfulness compared with similar interventions but without contact with nature $(k=7)$. The overall combined psychological, physiological, and interpersonal effects from pre- to post-intervention were statistically significant and of medium size $(g=0.54$, $p<0.001)$. Moderation analyses showed that natural environments characterized as forests/wild nature obtained larger numerical effects than environments characterized as gardens/parks, as did informal mindfulness compared with formal mindfulness. The small number of studies included, as well as the heterogeneity and generally low quality of the studies, must be taken into consideration when the results are interpreted. PROSPERO registration number: CRD42017065639.
\end{abstract}

Keywords: nature contact; mindfulness; meditation; nature-based therapy; intervention

\section{Introduction}

Recent research has paid increasing attention to the healing effects of both mindfulness training and exposure to nature, and research at the intersection between environmental psychology and meditation science seems to be a growing field [1], as does the variety of applications available [2]. Possible cumulative or synergistic effects may exist. An example is the correlation between the feeling of being connected to nature and the capacity to be mindful (i.e., trait mindfulness), which has been documented by Schutte and Malouff [3]. Another example is the theory-based hypothesis that meditation training and exposure to nature complement each other when combined [4], and are not merely the addition of independent effects. 


\subsection{Mindfulness}

Mindfulness is considered to involve the intentional regulation of attention with an attitude of non-judgment, openness, curiosity, and acceptance of one's current experience [5]. While state mindfulness may change rapidly, trait mindfulness can be enhanced through meditation. In programs such as mindfulness-based stress reduction (MBSR) and mindfulness-based cognitive therapy (MBCT), most formal meditations can be characterized as "attention training": Attentional regulation is cultivated by intentionally bringing the attention back to a chosen object (concentration meditation), by paying attention to whatever thoughts or sensations unfold by themselves in the mind (open-awareness meditation), or by being aware of the awareness itself [6,7]. Informal mindfulness is also part of the MBSR curriculum, and is cultivated while one engages in everyday activities [6]. Short mindfulness interventions have been documented to enhance state mindfulness [8] but most research on mindfulness training concerns the effects of programs such as the eight-week MBSR and MBCT. These have been proven to affect health positively with regard to psychological $[9,10]$, physical [11,12], and social functioning $[13,14]$. With regard to the mechanisms that underlie mindfulness interventions comparable to MBSR, evidence has been found regarding cognitive and emotional reactivity, mindfulness, and ruminative negative thinking, and preliminary but insufficient evidence has been found regarding self-compassion and psychological flexibility [15].

\subsection{Natural Environments and Exposure to Nature}

Nature can be defined as "areas containing elements of living systems that include plants and nonhuman animals across a range of scales and degrees of human management, from a small urban park through to relatively 'pristine wilderness'" [16] (p. 120). For the purposes of data extraction, this article uses Mausner's [17] categories of natural environments, which seem appropriate for the task and compatible with Bratman et al.'s definition.

It has been shown that spending time in nature promotes health, prevents health problems such as stress, depression, and anxiety [18-20], and improves immune functioning [21] and interpersonal functioning [22]. The pathways to positive health effects may be via physical and social activity as well as improved air quality [23]. Improved immune functioning from contact with microbial or other antigens [21] or exposure to certain natural substances such as phytoncides from trees [24] may also function as a mechanism for associated health benefits. The most widely investigated psychological pathways are attention restoration and stress reduction, but other factors, such as emotion regulation and feelings of awe or mystery, may mediate positive health outcomes [20]. A number of nature-based interventions have been developed that draw on the healing effects believed to be inherent to nature, including forest therapy, e.g., [25,26], wilderness therapy e.g., [27], adventure therapy e.g., [28], and horticultural therapy e.g., [29].

\subsection{Possible Interactions between Mindfulness and Exposure to Nature}

Attention is a psychological mechanism suggested to underlie the positive effects of both mindfulness training as a component of trait mindfulness [30], and exposure to nature [31] as part of attention restoration. Kaplan's attention restoration theory (ART) is one of the most widely applied and empirically supported theories about the benefits of exposure to nature for mental health [31-33]. According to ART, directed attention is a limited resource, but it may nonetheless be restored. ART suggests that exposure to nature can support the restorative process [34,35], in part because such natural settings are often physically distant from one's stressful everyday life, and also because nature promotes so-called soft fascination, which is defined as effortless attention drawn to fascinating objects. Although several environments and settings might potentially foster soft fascination, it has been suggested that natural environments are particularly well suited, as they inherently possess patterns that are sufficiently extended, rich, and coherent to engage the mind, which is believed to enable fascination in an "undramatic fashion" [35] (p. 174). ART is most often applied in relation to 
human-nature contact, but Kaplan [4] argues that similar mental processes may also occur during mindfulness meditation. The meditator is often distanced physically or mentally from everyday life, and can become softly fascinated as he or she effortlessly observes the stream of sensations, feelings, and thoughts. In order to be restorative, Kaplan argues that both exposure to nature and meditation need to fit the individual's inclinations, motivations, and capabilities [4]. He suggests in particular that the untrained meditator, who tends to use effort in meditation, will have easier access to soft fascination in a restorative environment [4]. This may be true whether the meditation is part of a manual-based mindfulness program or a single guided meditation.

Another possible interaction between attention restoration and mindfulness is that paying attention to the environment is necessary for soft fascination to occur. This was shown in a study by Jiang, Schmillen, and Sullivan [36]: Students in a natural environment who were occupied with their portable electronic devices did not restore their attention in the same way as students in the same natural environment with no such devices. However, even when one is willing to pay attention to nature, the mind can easily wander, and the recollection of present-moment awareness may be challenging. This capacity is cultivated through mindfulness, which would therefore seem to support the acquisition of the potential health benefits of exposure to nature.

As research including both mindfulness and nature is a growing field, and common mechanisms and interactions seem to exist, it may be timely to make an initial synthesis in order to assess the quality and extent of research in the field. To our knowledge, no systematic review or meta-analysis currently exists that investigates the potential effects of nature-based mindfulness (mindfulness conducted in nature).

\subsection{Aims}

The primary aim of this study was to systematically review all existing studies investigating nature-based mindfulness interventions, and to quantify the results through meta-analyses. The hypothesis was that, due to the beneficial health-promoting effects of mindfulness and exposure to nature, combinations of mindfulness and nature evaluated in open trials would be positive, and the effects of nature-based mindfulness would be superior to passive control conditions, to mindfulness in non-natural settings, and to interventions in natural settings without mindfulness. Specifically, manual-based stand-alone mindfulness conducted in nature was to be evaluated, with the hypothesis that exposure to nature would positively affect the outcomes. To qualify the results, the additional aims were to explore the potential moderating effect of 1 ) the type of natural setting and 2) the type of mindfulness practice.

\section{Materials and Methods}

The study was preregistered in PROSPERO (registration number CRD42017065639).

\subsection{Selection Criteria}

The PICOS approach [37] was used to evaluate studies' eligibility.

\subsubsection{Population}

Adults and adolescents ( $>12$ years) with or without a formal mental or physical diagnosis were included.

\subsubsection{Intervention}

Interventions included both exposure to nature as defined by Mausner [17] and guided mindfulness, defined as "paying attention on purpose, in the present moment." We left out one criterion often employed in the definition of mindfulness - namely, obtaining a non-judgmental attitude-in order not to exclude studies that did not address this more meta-cognitive component of the mindfulness construct cf. [38,39]. Studies were excluded if they only examined the effect of exposure to virtual or indoor nature. 


\subsubsection{Comparators}

Study groups were compared with (1) interventions without exposure to nature but with guided mindfulness, or (2) non-active control conditions (e.g., waiting lists or written materials). Studies were also included if they employed other or no comparators, in which case they were then evaluated as open trials.

\subsubsection{Outcomes}

These were any psychological (e.g., depression), physiological (e.g., heart rate), or interpersonal (e.g., prosocial orientation) outcomes based on client-level data for which an effect size could be calculated.

\subsubsection{Study Design}

Both open and controlled studies were evaluated. Only quantitative peer-reviewed studies reported in the English language were considered for inclusion.

\subsection{Search Strategy}

Search terms for mindfulness and nature were found in the target research articles. An abstract-based search was then conducted in electronic databases covering the natural environment and psychology. The databases searched were PsycINFO, Scopus, Web of Science, and Ovid, covering Agricola 1970 to August 2018; Ovid MEDLINE(R) Epub ahead of print, in-process, and other non-indexed citations; Ovid MEDLINE(R) Daily; Ovid MEDLINE(R) 1946 to present; Cab Abstracts 1910-2018; and Embase 1974 to present. Search terms related to mindfulness (Meditati* or mindfulness or MBSR or MBCT) were combined with search terms related to therapies used in natural settings—or "forest bathing", Ecotherapy, "Eco therapy", "Eco-therapy", "Nature-based therapy", "Nature-based therapy", "Wilderness therapy", "Horticultural therapy", "Nature therapy", "Nature involvement", "nature-based intervention", " cognitive behavior therapy", or "Nature-assisted therapy" —and with search terms related to the natural environment and "Restorative nature", "nature contact", "nature exposure", "nature-based activities", "Restorative garden", "Healing nature", "Healing garden", "Therapeutic nature", "Therapeutic garden", "Therapy garden", “Care garden*”, Wilderness, Forest*, Woods, Outdoor*, “Open space*”, Park, “Green space ${ }^{* \prime}$, greenspace, "Natural environment", “Marine environment", “Ocean wealth", or "Blue gym".

The search terms were defined and the searches were conducted for the period from the earliest dates available in the databases through August 2018. Additionally, a backward search (snowballing) and a forward search (citation-tracking) were conducted for the included articles. Abstracts and full texts were evaluated, and disagreements over the inclusion/exclusion of a study were resolved by consensus.

\subsection{Data Extraction}

Means and standard deviations for all quantitative outcomes were extracted. When these were not available, other test parameters were used (e.g., $F$ and $p$ ). In cases where an effect size could not be calculated, the study's authors were contacted. Studies were coded for participant characteristics (i.e., age, gender, and race/ethnicity), duration of intervention (from first to last intervention session), time to follow-up, and number of hours with mindfulness practice. All health outcomes were categorized as measures of physical, psychological, or interpersonal outcomes. The characteristics of nature were coded as either mixed outdoor environment containing natural elements (often with predominant built structures), garden/park with settings composed of natural elements intended to make it "appear natural", or forest/wild nature with predominantly natural elements unaffected by human interventions [17]. The types of mindfulness were coded as formal mindfulness i.e., guided meditation, or informal mindfulness where attention is guided to the present moment during 
everyday activities. Formal mindfulness was coded as open-awareness meditation versus concentration meditation. Mindfulness was also coded as the intention to induce state mindfulness or build trait mindfulness [6,7]. All the coding was verified by the co-author. Data extraction protocols are available upon request from the corresponding author.

\subsection{Quality Assessment}

Studies were evaluated for quality using the quality assessment tool for quantitative studies from the Effective Public Health Practice Project (EPHPP) [40]. For each of the six components: Selection bias, study design, confounders, blinding, data collection methods, and withdrawals and dropouts, the studies were rated as strong, moderate, or weak, following the guidelines from the EPHPP tool. These ratings were, also in accordance with the EPHPP guidelines summed to create a global quality score (see Table 1). For studies with no weak ratings for the six components, the global quality score was set to be $1=$ strong. Studies with one weak rating the summed score was set to be $2=$ moderate. If two or more of the six components had weak scores, the global quality rating was set to be $3=$ weak. Ratings were made and disagreements were discussed and resolved by consensus.

\subsection{Analytical Overview}

Meta-analyses were performed for the designs and outcomes combined, as well as separately for each of the design types and each of the outcome categories. Analyses were conducted for two time periods: From pre- to immediately post-intervention, and from pre-intervention to the last follow-up assessment. All analyses were based on random-effects models.

The planned exploratory moderation analyses of categorical variables (e.g., type of nature and type of mindfulness) were explored with meta-ANOVAs. Analyses were performed when a sufficient number of studies $(k \geq 3)$ was identified for a given moderator category. Continuous moderator variables (i.e., age, $\%$ women, $\%$ Whites, number of sessions with mindfulness, duration of treatment, and time to last follow-up) were considered in meta-regression analyses, based on random-effects models and estimated with the maximum likelihood method. In the regression analyses, the proposed moderators served as independent variables, with effect size serving as the dependent variable.

Effect sizes were expressed as Hedges' $g$ in order to adjust for potential bias to overestimate the effect size in small samples [41], with values of $0.2,0.5$, and 0.8 considered small, medium, and large respectively [42]. A $p$-value below 0.05 was considered statistically significant. Positive effect sizes indicate a positive effect of the interventions. Each effect size was weighted by its precision (inverse variance), so that interventions with larger samples contributed more to the estimate of the overall effect size. Heterogeneity was explored using $Q$ and $I^{2}$ statistics. Q-tests concern the probability that results reflect systematic between-study differences. Due to the generally low statistical power of heterogeneity tests, a $p$-value of 0.10 was used to indicate heterogeneity [43]. The $\mathrm{I}^{2}$ statistic is an estimate of the degree of heterogeneity, and is considered to be unaffected by the number of studies. An $\mathrm{I}^{2}$ value of $0 \%$ indicates no observed heterogeneity. Values of $25 \%, 50 \%$, and $75 \%$ are considered low, moderate, and high respectively [44].

Positive and negative findings may not be equally likely to get published, introducing the risk of publication bias. The distribution of effect sizes was therefore visually inspected by means of funnel plots and tested with Egger's test [45]. When the analyses indicated possible publication bias, an adjusted effect size was estimated using Duval and Tweedie's [46] trim-and-fill method, which imputes missing results and recalculates the effect size. In addition, the file drawer problem — the possibility that unidentified or unpublished studies with null findings could alter statistically significant meta-analysis results-was evaluated by Rosenthal's fail-safe number [47]. If the fail-safe number exceeded $5 \mathrm{k}+10$, with $\mathrm{k}$ being the number of studies included in the meta-analysis, the file drawer problem was considered sufficiently low to allow acceptance of the results as unaffected by that potential source of bias.

All analyses were conducted using the Comprehensive Meta-Analysis program, version 3.3.070 Eaglewood, NJ, USA: Biostat (“Comprehensive Meta-Analysis," 2014). 


\section{Results}

\subsection{Search Results}

A total of 987 publications were identified: 949 through searches in databases, and 38 from other sources. After the screening of abstracts, 841 records were excluded, resulting in 146 full-text articles; of these, 120 were excluded, primarily due to a lack of relevant content on interventions (see Figure 1). Five authors were contacted for the data necessary to calculate an effect size. Three authors provided the data. In the fourth case, the effect size was set to zero, and the study was included. The last study was excluded due to a lack of information concerning the relevant outcomes. In total, 26 articles from 25 independent studies were included. Seven interventions with mindfulness in a natural setting were compared with a similar intervention without exposure to nature (i.e., active control). Seven studies compared interventions with groups in non-active control conditions (i.e., passive control), and 13 studies were evaluated as open trials.

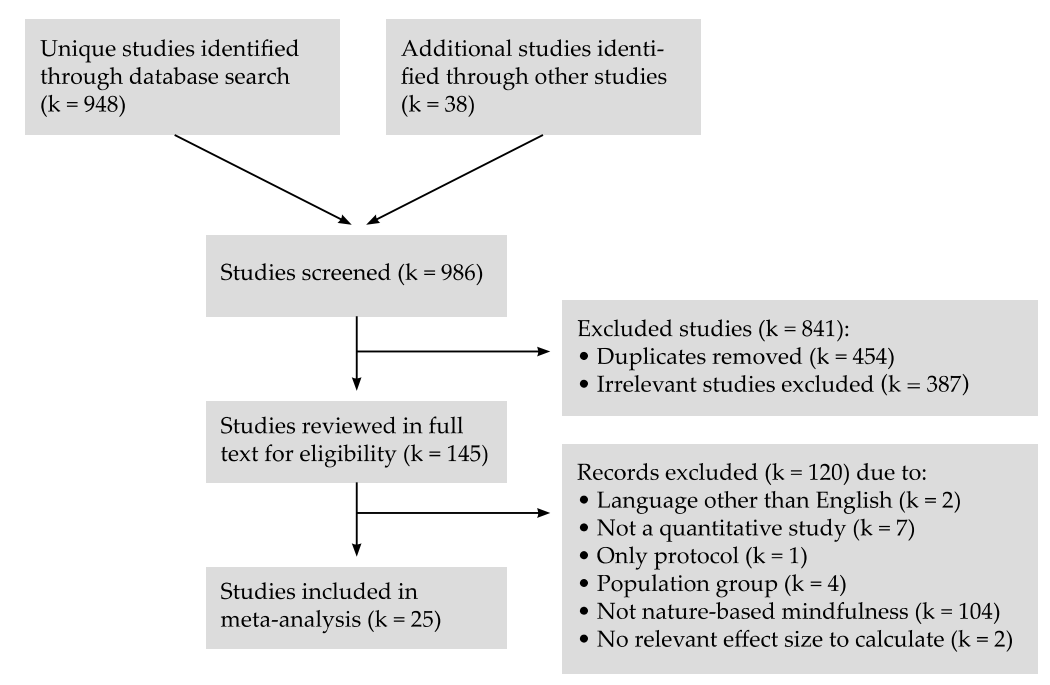

Figure 1. Flow chart of literature search.

\subsection{Characteristics of Studies}

The characteristics of the 25 included studies are summarized in Table 1. Most studies were from Western countries (12 North American and five European), while eight were from Southeast Asia. Included in the meta-analysis were a total of 2990 participants across the studies (mean $N$ per study: 120, range 19-659). The mean age was 30.71 (range 12-89), with $51.8 \%$ female (range $0-100$ ) and $66.2 \%$ White/Caucasian participants. In five studies, physical illnesses were targeted (i.e., cancer, hypertension, coronary diseases, chronic diseases, or pain). In six studies the treatment target was a psychological diagnosis (e.g., stress, anxiety, and depression), and five studies investigated treatment for substance abuse. Eight included participants with no diagnoses. Twelve studies reported effects on psychological measures (e.g., anxiety and depression). Nine studies had physical outcome measures (e.g., heart rate variability and cortisol level), and eight studies reported effects on interpersonal measures (e.g., work function).

The EPHPP assessment tool was used to evaluate the studies' quality [40]. Global ratings were strong for one study, fair for six, and weak for 18 studies. Most studies obtained a strong score for data collection methods $(\mathrm{k}=22)$ and for reporting withdrawals and dropouts $(\mathrm{k}=14)$; on the other hand, most studies obtained a weak score for selection bias $(\mathrm{k}=13)$ and blinding $(\mathrm{k}=21)$. 
Table 1. Characteristics of included studies.

\begin{tabular}{|c|c|c|c|c|c|c|c|}
\hline $\begin{array}{l}\text { Study } \\
\text { Author, Year, Country }\end{array}$ & $\begin{array}{c}\text { Population } \\
N=\text { Included } \\
\text { (Completed/Follow-up), } \\
\text { Age = Range (Mean), Gender, } \\
\text { Ethnicity, Target Group }\end{array}$ & $\begin{array}{c}\text { Intervention } \\
\text { Duration, Content }\end{array}$ & $\begin{array}{c}\text { Comparators Duration, } \\
\text { Content }\end{array}$ & $\begin{array}{c}\text { Outcomes } \\
\text { Measurements, Tools }\end{array}$ & $\begin{array}{c}\text { Time } \\
\text { Intervention, Timings of } \\
\text { Data Collection }\end{array}$ & $\begin{array}{c}\text { Setting } \\
\text { Location of Study Group }\end{array}$ & $\begin{array}{l}\text { EPHPP } \\
\text { Quality }\end{array}$ \\
\hline \multicolumn{8}{|c|}{ Comparator: interventions incl. mindfulness, but without nature contact } \\
\hline $\begin{array}{l}\text { Ballew \& Omoto } \\
\text { (2018) [48] } \\
\text { USA }\end{array}$ & $\begin{array}{c}N=100 \\
\text { Age }=18-24(19.3) \\
\% \text { female }=55 \\
\% \text { Whites }=21 \\
\text { Study group: Students, no diagnosis }\end{array}$ & $\begin{array}{l}15 \mathrm{~min} \text { in natural settings. } \\
\text { Instructions to look at surrounding } \\
\text { features and pay attention to all } \\
\text { details, colors, and textures, to use all } \\
\text { senses to take everything in. } \\
\text { Note-taking. }\end{array}$ & $\begin{array}{l}\text { Same program as study } \\
\text { group, human-built outdoor } \\
\text { environment. }\end{array}$ & $\begin{array}{l}\text { Absorption, awe, happiness, } \\
\text { joy, contentment (rating } \\
\text { sentences) }\end{array}$ & $\begin{array}{l}\text { T1: Survey just after 15-min. } \\
\text { intervention }\end{array}$ & $\begin{array}{l}\text { Arboretum, sitting on a } \\
\text { bench, view of trees, creek, } \\
\text { bamboo, etc. }\end{array}$ & 3 \\
\hline $\begin{array}{l}\text { Shin et al. } \\
\text { (2012) [49] } \\
\text { Korea }\end{array}$ & $\begin{array}{c}N=69(68) \\
\text { Age }=20.4( \pm 1.5) \\
\% \text { female }=100 \\
\% \text { Whites }=\text { N/A } \\
\text { Study group: Students, no diagnosis }\end{array}$ & $\begin{array}{l}35 \mathrm{~min} \text {. walk, } 10 \mathrm{~min} \text {. rest, } 35 \mathrm{~min} \text {. } \\
\text { walk, } 10 \mathrm{~min} \text {. rest. Mindful walking } \\
\text { with focus on breath and sensations. }\end{array}$ & $\begin{array}{l}\text { Same program as study } \\
\text { group, indoor setting. }\end{array}$ & $\begin{array}{l}\text { Anxiety (STAI), self-esteem } \\
\text { (RSE), happiness (HI-K) }\end{array}$ & $\begin{array}{l}\text { T1: Before intervention } \\
\text { T2: Just after 90-min. } \\
\text { intervention }\end{array}$ & $\begin{array}{c}\text { Undisturbed rocky area } \\
\text { with old-growth } \\
\text { broad-leaved evergreen } \\
\text { trees }\end{array}$ & 3 \\
\hline $\begin{array}{l}\text { Passmore \& Holder [50] } \\
\text { (2017) } \\
\text { Canada }\end{array}$ & $\begin{array}{c}N=395(364) \\
\text { Age }=20.09 \\
\% \text { female }=67 \\
\% \text { Whites }=\text { N/A } \\
\text { Study group: Students, no diagnosis }\end{array}$ & $\begin{array}{l}2 \text { weeks. Instructions to be mindful } \\
\text { of emotions evoked by natural } \\
\text { objects/scenes in everyday life; to } \\
\text { describe strong emotions and take } \\
\text { pictures of the nature that evoked } \\
\text { them. }\end{array}$ & $\begin{array}{l}\text { Same intervention as study } \\
\text { group, human built } \\
\text { environment. } \\
\text { Passive controls: Continue } \\
\text { regular routines. }\end{array}$ & $\begin{array}{l}\text { Affect (PANAS), elevation } \\
\text { (EES), meaning (SMS), } \\
\text { connectedness (GSC), } \\
\text { prosocial orientation (PSO), } \\
\text { connectedness to nature } \\
\text { (CNS), engaging with } \\
\text { beauty (EWB) }\end{array}$ & $\begin{array}{l}\text { T1: Just before intervention } \\
\text { (PANAS only) } \\
\text { T2: Just after 2-week } \\
\text { intervention (all } \\
\text { measurements) }\end{array}$ & $\begin{array}{l}\text { Everyday environment of } \\
\text { university students }\end{array}$ & 3 \\
\hline $\begin{array}{l}\text { Kim et al. } \\
\text { (2009) [51] } \\
\text { Korea }\end{array}$ & $\begin{array}{c}N=73(63) \\
\text { Age }=46.2 \\
\% \text { female }=85.7 \\
\% \text { Whites }=\text { N/A } \\
\text { Study group: Major depressive } \\
\text { disorder }\end{array}$ & $\begin{array}{c}4 \text { weeks, } 3 \text { hours weekly, for } \mathrm{CBT} \text {, } \\
\text { positive psychology tools, and } \\
\text { mindfulness meditation on breath, } \\
\text { wind, forest, and sounds (insight } \\
\text { meditation). }\end{array}$ & $\begin{array}{l}\text { Same program as study } \\
\text { group, indoor setting. } \\
\text { Meditation focus on breath } \\
\text { and indoor or window } \\
\text { objects. } \\
\text { Passive control: TAU. }\end{array}$ & $\begin{array}{l}\text { Depression (BDI, HRSD, } \\
\text { MADRS), quality of life } \\
\text { (SF-36), stress (HRV, } \\
\text { cortisol) }\end{array}$ & $\begin{array}{l}\text { T1: Just before treatment, all } \\
\text { measurements } \\
\text { T2: T1 + } 1 \text { week, depression } \\
\text { questionnaires } \\
\text { T3: T1 }+2 \text { weeks, } \\
\text { depression questionnaires } \\
\text { T4: After } 3 \text { weeks of } \\
\text { treatment, all measurements }\end{array}$ & $\begin{array}{l}\text { 44-ha arboretum, } 2035 \\
\text { species }\end{array}$ & 2 \\
\hline $\begin{array}{l}\text { Willert et al. (2014) [52] } \\
\text { Denmark }\end{array}$ & $\begin{array}{c}N=93(66 / 49) \\
\text { Age }=25-59(45.0) \\
\% \text { female }=82.8 \\
\% \text { Whites }=\text { N/A } \\
\text { Study group: Stressed students }\end{array}$ & $\begin{array}{l}16 \text { weeks, } 5 \text { days a week, } 9 \text { a.m. to } \\
\text { afternoon. Groups of max. } 12 \text {. } \\
\text { All-day exercises from meditation } \\
\text { training programs (MBCT and Five } \\
\text { Tibetans), horticultural activities, } \\
\text { nature walks, stress management, job } \\
\text { counseling, individual } \\
\text { psychotherapy sessions. }\end{array}$ & $\begin{array}{l}\text { Same program as study } \\
\text { group, indoor setting. }\end{array}$ & $\begin{array}{c}\text { Stress (PSS-10), sleep } \\
\text { (BNSQ), mindfulness } \\
\text { (FFMQ - } 3 \text { dimensions), } \\
\text { self-efficacy (COPSOQ-II), } \\
\text { Outcome Rating Scale, work } \\
\text { ability (WAI) }\end{array}$ & $\begin{array}{l}\text { T1: Just before treatment } \\
\text { T2: Just after } 3 \text { months of } \\
\text { treatment } \\
\text { T3: T2 + } 3 \text { months }\end{array}$ & $\begin{array}{l}\text { Garden incl. greenhouse, } \\
\text { near forest and beach }\end{array}$ & 2 \\
\hline $\begin{array}{l}\text { Vujcic et al. (2017) [53] } \\
\text { Serbia }\end{array}$ & $\begin{array}{c}N=30 \\
\text { Age }=25-65(45.35) \\
\% \text { female }=70 \\
\% \text { Whites }=\text { N/A } \\
\text { Study group: Depressed, anxious }\end{array}$ & $\begin{array}{l}4 \text { weeks, } 3 \text { one-hour sessions per } \\
\text { week of horticultural therapy, art } \\
\text { therapy, and relaxation/meditation } \\
\text { sessions. All main activities relate to } \\
\text { work with living plants. }\end{array}$ & $\begin{array}{l}\text { Parallel indoor activities } \\
\text { with study group, incl. } \\
\text { occupational, art, and } \\
\text { conventional therapies. }\end{array}$ & $\begin{array}{l}\text { Depression and anxiety } \\
\text { (DASS21) }\end{array}$ & $\begin{array}{l}\text { T1: Just before intervention } \\
\text { T2: Just after } 4 \text { weeks' } \\
\text { intervention }\end{array}$ & $\begin{array}{l}\text { Botanical garden incl. open } \\
\text { space, greenhouse, Japanese } \\
\text { garden, fountain }\end{array}$ & 3 \\
\hline
\end{tabular}


Table 1. Cont.

\begin{tabular}{|c|c|c|c|c|c|c|c|}
\hline $\begin{array}{l}\text { Study } \\
\text { Author, Year, Country }\end{array}$ & $\begin{array}{c}\text { Population } \\
N=\text { Included } \\
\text { (Completed/Follow-up), } \\
\text { Age = Range (Mean), Gender, } \\
\text { Ethnicity, Target Group }\end{array}$ & $\begin{array}{c}\text { Intervention } \\
\text { Duration, Content }\end{array}$ & $\begin{array}{c}\text { Comparators Duration, } \\
\text { Content }\end{array}$ & $\begin{array}{c}\text { Outcomes } \\
\text { Measurements, Tools }\end{array}$ & $\begin{array}{c}\text { Time } \\
\text { Intervention, Timings of } \\
\text { Data Collection }\end{array}$ & $\begin{array}{c}\text { Setting } \\
\text { Location of Study Group }\end{array}$ & $\begin{array}{l}\text { EPHPP } \\
\text { Quality }\end{array}$ \\
\hline $\begin{array}{l}\text { Lymeus } \\
\text { (2018) [54] } \\
\text { Sweden }\end{array}$ & $\begin{array}{c}\text { Study 1: } \\
N=89 \\
\text { Age }=(23) \\
\% \text { female }=64 \\
\% \text { Whites }=\text { N/A } \\
\text { Study 2: } \\
N=51 \\
\text { Age }=(23) \\
\text { \% female }=72.5 \\
\text { \% Whites }=\text { N/A } \\
\text { Study groups: Stressed }\end{array}$ & $\begin{array}{l}5 \text { weeks, } 1 \text { weekly 90-min. session. } \\
\text { Manual-based meditation training } \\
\text { program (REST), each session } \\
\text { beginning and ending with } 15-20 \\
\text { min. guided open monitoring } \\
\text { meditation (no specific tradition). } \\
\text { Exercises and themes. Homework: } \\
15 \text { min. meditation most days. }\end{array}$ & $\begin{array}{l}\text { Classroom setting. Same } \\
\text { schedule as study group. } \\
\text { Beginning and end of } \\
\text { sessions: Focused attention } \\
\text { meditation (no specific } \\
\text { tradition), exercises and } \\
\text { themes. }\end{array}$ & $\begin{array}{l}\text { Attention (LDST; TMT } \\
\text { study 2), affect (SCAS) }\end{array}$ & $\begin{array}{c}\text { Study 1: } \\
\text { T1: Before/after session } 1 \\
\text { T2: Before/after session } 3 \\
\text { T3: Before/after session } 5 \\
\text { Study 2: } \\
\text { T1: Enrollment } \\
\text { T2: Before/after initial } \\
\text { 20-min. meditation in } \\
\text { session } 1 \\
\text { T3: Before/fter initial } \\
\text { 20-min. meditation in } \\
\text { session } 3 \\
\text { T4: Before/after initial } \\
\text { 20-min. meditation in } \\
\text { session } 5\end{array}$ & $\begin{array}{l}\text { Botanical garden incl. } \\
\text { tropical greenhouse, water } \\
\text { bodies, orangery }\end{array}$ & 3 \\
\hline \multicolumn{8}{|c|}{ Comparator: Non-active control conditions (see also Kim 2009 and Passmore 2017 above) } \\
\hline $\begin{array}{l}\text { Han } \\
\text { (2016) [55] } \\
\text { Korea }\end{array}$ & $\begin{array}{c}N=61 \\
\text { Age }=25-49(39.75) \\
\% \text { female }=57.4 \\
\% \text { Whites }=\text { N/A } \\
\text { Study group: Chronic pain }\end{array}$ & $\begin{array}{l}\text { 24-hour residential intervention } \\
\text { (noon to noon). In forest: Walking, } \\
\text { therapeutic activities, physical } \\
\text { exercises, mindfulness meditation. } \\
\text { Indoor music therapy, } \\
\text { psycho-education on stress and pain. }\end{array}$ & $\begin{array}{l}\text { Usual weekend routines, } \\
\text { except visiting natural } \\
\text { environment or heavy loads } \\
\text { of work. }\end{array}$ & $\begin{array}{l}\text { Stress (HRV, HR) natural } \\
\text { killer cells (NK), pain (VAS), } \\
\text { depression (BDI), } \\
\text { health-related quality of life } \\
\text { (EQ-VAS) }\end{array}$ & $\begin{array}{l}\text { T1: Just before treatment } \\
\text { T2: T1 + } 1 \text { day just after } 24 \\
\text { hours' treatment }\end{array}$ & $\begin{array}{l}\text { Foot of a mountain: forest } \\
\text { valley, "spectacular" views }\end{array}$ & 3 \\
\hline $\begin{array}{l}\text { Won } \\
\text { (2012) [56] } \\
\text { Korea }\end{array}$ & $\begin{array}{c}N=92 \\
\text { Age }=45.26 \\
\% \text { female }=8.7 \\
\% \text { Whites }=\text { N/A } \\
\text { Study group: Detoxified chronic } \\
\text { alcoholics }\end{array}$ & $\begin{array}{l}9 \text { days: } 3 \text { days actively interacting } \\
\text { with nature, } 3 \text { days challenging } \\
\text { activities in nature, } 3 \text { days activities } \\
\text { for introspection (nature meditation, } \\
\text { counseling in nature etc.). }\end{array}$ & $\begin{array}{l}\text { Inpatients, no specific } \\
\text { treatment described. }\end{array}$ & Depression (BDI) & $\begin{array}{l}\text { T1: Just before treatment } \\
\text { T2: T1 + } 9 \text { days just after } \\
\text { treatment }\end{array}$ & 2140-ha recreational forest & 2 \\
\hline $\begin{array}{l}\text { Warber et al. (2011) [57] } \\
\text { USA }\end{array}$ & $\begin{array}{c}N=58(47 / 41) \\
\text { Age }=25-75(59.3) \\
\% \text { female }=40.4 \\
\% \text { Whites }=85.1 \\
\text { Study group: Coronary syndrome }\end{array}$ & $\begin{array}{l}\text { 4-day residential program. } \\
\text { Study group } 1 \text { (MFTE): Meditation, } \\
\text { guided imagination, journaling, } \\
\text { drawing, nature activities, nature } \\
\text { imagination. } \\
\text { Study group 2 (LCP): Nutrition, } \\
\text { physical exercise, stress management } \\
\text { based on mindfulness and } \\
\text { whole-person approach. } \\
\text { Both groups: Telephone coaching } \\
\text { biweekly for } 3 \text { months. }\end{array}$ & No treatment. & $\begin{array}{c}\text { Depression (BDI, BSI), stress } \\
\text { (PSS), hope (SHS), gratitude } \\
\text { (SG), quality of life (SF-36), } \\
\text { spirituality (ISWBS), } \\
\text { personal transformation } \\
\text { (TCQ), physical activity } \\
\text { (PPAQ), } \\
\text { stress (HR, BP, BMI, lipid } \\
\text { levels, lipid particle size, } \\
\text { high sensitivity C-reactive } \\
\text { protein, biomarkers IL-6 } \\
\text { and IL-10) }\end{array}$ & $\begin{array}{l}\text { T1: Just before treatment } \\
\text { T2: Just after } 4 \text { days' } \\
\text { treatment } \\
\text { T3: T2 }+3 \text { months } \\
\text { T4: T2 }+6 \text { months } \\
\text { Biophysical measurements } \\
\text { only at T1 and T2 }\end{array}$ & "Beautiful" rural settings & 3 \\
\hline
\end{tabular}


Table 1. Cont.

\begin{tabular}{|c|c|c|c|c|c|c|c|}
\hline $\begin{array}{l}\text { Study } \\
\text { Author, Year, Country }\end{array}$ & $\begin{array}{c}\text { Population } \\
N=\text { Included } \\
\text { (Completed/Follow-up), } \\
\text { Age = Range (Mean), Gender, } \\
\text { Ethnicity, Target Group }\end{array}$ & $\begin{array}{c}\text { Intervention } \\
\text { Duration, Content }\end{array}$ & $\begin{array}{c}\text { Comparators Duration, } \\
\text { Content }\end{array}$ & $\begin{array}{c}\text { Outcomes } \\
\text { Measurements, Tools }\end{array}$ & $\begin{array}{c}\text { Time } \\
\text { Intervention, Timings of } \\
\text { Data Collection }\end{array}$ & $\begin{array}{c}\text { Setting } \\
\text { Location of Study Group }\end{array}$ & $\begin{array}{l}\text { EPHPP } \\
\text { Quality }\end{array}$ \\
\hline $\begin{array}{l}\text { Sung et al. } \\
(2012)[58] \\
\text { Korea }\end{array}$ & $\begin{array}{c}N=56 \\
\text { Age }=66.0 \\
\% \text { female }=60.7 \\
\% \text { Whites }=\text { N/A } \\
\text { Study group: Hypertension stage 1 }\end{array}$ & $\begin{array}{l}\text { 3-day forest therapy program: } \\
\text { Relationship-building, stress and } \\
\text { health management, mindfulness } \\
\text { and gratitude meditation in forest. }\end{array}$ & $\begin{array}{c}\text { Written material on } \\
\text { hypertension management. }\end{array}$ & $\begin{array}{c}\text { Stress (BP), salivary cortisol } \\
\text { level, quality of life (QoL, } 5 \\
\text { dimensions) }\end{array}$ & $\begin{array}{l}\text { T1: Just before intervention, } \\
\text { all measurements } \\
\text { T2: Just after 3-day } \\
\text { intervention, all } \\
\text { measurements } \\
\text { Follow-up: Self- monitoring } \\
\text { BP, 8 weeks }\end{array}$ & $\begin{array}{l}\text { Recreational forest in } \\
\text { mountain region }\end{array}$ & 2 \\
\hline $\begin{array}{l}\text { Passmore } \\
\text { (2014) [59] } \\
\text { Canada }\end{array}$ & $\begin{array}{c}N=86(84) \\
\text { Age }=18-45(20.96) \\
\% \text { female }=86.9 \\
\% \text { Whites }=\text { N/A } \\
\text { Study group: Students, no diagnosis }\end{array}$ & $\begin{array}{l}14 \text { days. Written instructions to } \\
\text { immerse themselves in nature } \\
\text { activities whenever possible in } \\
\text { everyday lives. Keeping logbook of } \\
\text { nature activities for each day. }\end{array}$ & $\begin{array}{l}\text { Solving anagram puzzles } \\
\text { whenever possible in their } \\
\text { everyday lives. }\end{array}$ & $\begin{array}{l}\text { Affect (PANAS), elevation } \\
\text { (EES), meaning (SMS), } \\
\text { motivation (SCM) }\end{array}$ & $\begin{array}{l}\text { T1: Just before intervention } \\
\text { T2: T1 + } 14 \text { days just after } \\
\text { intervention }\end{array}$ & $\begin{array}{l}\text { Everyday environment of } \\
\text { Canadian students }\end{array}$ & 1 \\
\hline \multicolumn{8}{|c|}{ Studies with no comparators relevant for this review } \\
\hline $\begin{array}{l}\text { Jung } \\
\text { (2015) [60] } \\
\text { Korea }\end{array}$ & $\begin{array}{c}N=19 \\
\text { Age }=29.4 \\
\% \text { female }=100 \\
\% \text { Whites }=\text { N/A } \\
\text { Study group: No diagnosis }\end{array}$ & $\begin{array}{l}2 \text { days, noon day } 1 \text { to noon day } 3 \text {. } \\
\text { Indoors: lectures on coping with } \\
\text { stress, counseling, cognitive therapy, } \\
\text { music therapy. In forest: } 5 \text { hours' } \\
\text { meditation, walking, exercises. }\end{array}$ & & $\begin{array}{c}\text { Stress (HR, HRV, cortisol), } \\
\text { natural killer cell activity } \\
\text { (NK), burnout (MBI-GS), } \\
\text { stress (WSRI), recovery } \\
\text { (REQ) }\end{array}$ & $\begin{array}{l}\text { T1: Just before intervention } \\
\text { T2: T1 + } 2 \text { days just after } \\
\text { intervention } \\
\text { T3: } 22+14 \text { days } \\
\text { All measures at T1 and T2, } \\
\text { except MBI-GS second } \\
\text { measure at T3 }\end{array}$ & 2140-ha recreational forest & 3 \\
\hline $\begin{array}{l}\text { Yu et al. } \\
\text { (2017) [61] } \\
\text { Taiwan }\end{array}$ & $\begin{array}{c}N=128 \\
\text { Age }=45-86(60) \\
\% \text { female }=65.6 \\
\% \text { Whites }=\text { N/A } \\
\text { Study group: } 46 \% \text { chronic diseases } \\
\text { (e.g., diabetes) }\end{array}$ & $\begin{array}{l}2 \text { hours, } 2.5 \mathrm{~km} \text { sensory forest walk } \\
\text { incl. guided stimulation of senses } \\
\text { (visual, auditory, olfactory, tactile). } \\
\text { Groups of 6-12 participants. }\end{array}$ & & $\begin{array}{c}\text { Mood (POMS-SF), anxiety } \\
\text { (STAI), stress (pulse rate, BP, } \\
\text { HR, HRV) }\end{array}$ & $\begin{array}{l}\text { T1: Just before intervention } \\
\text { T2: Just after 2-hour } \\
\text { intervention }\end{array}$ & $\begin{array}{l}\text { Sensory forest, in valley } \\
\text { surrounded by mountains } \\
\text { on three sides (part of Xitou } \\
\text { Nature Education Area in } \\
\text { Japan) }\end{array}$ & 3 \\
\hline $\begin{array}{l}\text { Korpela et al. (2017) [62] } \\
\text { Finland, Luxembourg, } \\
\text { Sweden }\end{array}$ & $\begin{array}{c}N=283 \\
\text { Age }=13-82(47.2) \\
\% \text { female }=74 \\
\% \text { Whites }=\text { N/A } \\
\text { Study group: No diagnosis }\end{array}$ & $\begin{array}{l}\text { Well-being trails in the } 3 \text { countries, } \\
4.4-6.6 \mathrm{~km} \text {, containing the same } 9 \\
\text { signposts with tasks: Self-monitoring } \\
\text { (first and last), relaxation, letting } \\
\text { oneself be fascinated, observing } \\
\text { nature and one's own body and } \\
\text { mood. }\end{array}$ & & $\begin{array}{l}\text { Restorative change ( } 4 \text { items), } \\
\text { mood ( } 1 \text { item), nature } \\
\text { connectedness ( } 3 \text { items) }\end{array}$ & $\begin{array}{l}\text { T1: At first signpost on the } \\
\text { trail } \\
\text { T2: At last signpost on the } \\
\text { trail }\end{array}$ & $\begin{array}{l}\text { Hiking tracks incl. forests, } \\
\text { lakesides, fields, cultural } \\
\text { landscapes }\end{array}$ & 3 \\
\hline $\begin{array}{l}\text { Yang } \\
\text { (2018) [63] } \\
\text { USA }\end{array}$ & $\begin{array}{c}\quad N=29(27 / 26) \\
\text { Age }=66-89(73.2) \\
\% \text { female }=83 \\
\% \text { Whites }=79 \\
\text { Study group: No diagnosis }\end{array}$ & $\begin{array}{l}4 \text { weeks, } 8 \text { sessions of } 30 \text { min. } \\
\text { individual mindful walking. Before } \\
\text { walking, guidance either to become } \\
\text { familiar with the environment, to } \\
\text { focus on breath or movement, or to } \\
\text { scan through the body. }\end{array}$ & & $\begin{array}{l}\text { Affect (PROMIS), } \\
\text { mindfulness (SMS) }\end{array}$ & $\begin{array}{l}\text { T1: Just before treatment } \\
\text { T2: Just after } 30 \mathrm{~min} . \\
\text { mindful walking }\end{array}$ & $\begin{array}{l}\text { Flat designated route in } \\
\text { arboretum }\end{array}$ & 2 \\
\hline
\end{tabular}


Table 1. Cont.

\begin{tabular}{|c|c|c|c|c|c|c|c|}
\hline $\begin{array}{l}\text { Study } \\
\text { Author, Year, Country }\end{array}$ & $\begin{array}{c}\text { Population } \\
N=\text { Included } \\
\text { (Completed/Follow-up), } \\
\text { Age = Range (Mean), Gender, } \\
\text { Ethnicity, Target Group }\end{array}$ & $\begin{array}{c}\text { Intervention } \\
\text { Duration, Content }\end{array}$ & $\begin{array}{c}\text { Comparators Duration, } \\
\text { Content }\end{array}$ & $\begin{array}{c}\text { Outcomes } \\
\text { Measurements, Tools }\end{array}$ & $\begin{array}{c}\text { Time } \\
\text { Intervention, Timings of } \\
\text { Data Collection }\end{array}$ & $\begin{array}{c}\text { Setting } \\
\text { Location of Study Group }\end{array}$ & $\begin{array}{l}\text { EPHPP } \\
\text { Quality }\end{array}$ \\
\hline $\begin{array}{l}\text { Corazon et al. (2018) [64] } \\
\text { \& Stigsdotter } \\
\text { (2018) [65] } \\
\text { Denmark }\end{array}$ & $\begin{array}{c}N=43(42 / 29) \\
\text { Age }=47.9 \\
\% \text { female }=81.6 \\
\% \text { Whites }=\text { N/A } \\
\text { Study group: Severely stressed }\end{array}$ & $\begin{array}{l}\text { 10-week nature-based therapy, } 3 \\
\text { times, } 3 \text { hours per week. Activities } \\
\text { individually and in groups: Exercises } \\
\text { in accordance with } \\
\text { MBSR and related to nature } \\
\text { experiences, such as mindful walking } \\
\text { in natural setting. Gardening and } \\
\text { relaxation/reflection time. Individual } \\
\text { therapeutic sessions (CBT) and } \\
\text { support for return to work. }\end{array}$ & & $\begin{array}{l}\text { Sick leave and contact with } \\
\text { GP (from national database), } \\
\text { well-being (PGWBI), } \\
\text { burnout (SMBQ) }\end{array}$ & $\begin{array}{c}\text { T0-T1: 1-year time span } \\
\text { T1: Treatment start } \\
\text { T2: Just after } 10 \text { weeks' } \\
\text { treatment } \\
\text { T3: T2 }+3 \text { months } \\
\text { T4: T2 + } 6 \text { months } \\
\text { T5: T2 + } 12 \text { months }\end{array}$ & $\begin{array}{l}\text { 1.4-ha wild forest garden } \\
\text { located in larger arboretum }\end{array}$ & 2 \\
\hline $\begin{array}{l}\text { Sahlin et al. } \\
\text { (2014) [66] } \\
\text { Sweden }\end{array}$ & $\begin{array}{c}N=44(33) \\
\text { Age = N/A } \\
\% \text { female = } 100 \\
\% \text { Whites }=\text { N/A } \\
\text { Study group: Stressed }\end{array}$ & $\begin{array}{l}12 \text { weeks, } 3 \text { hours weekly. } 3 \\
\text { intervention groups Activities: } \\
\text { Walks, relaxation, mindfulness, } \\
\text { therapeutic painting, group therapy, } \\
\text { information about stress and health, } \\
\text { garden and nature activities. }\end{array}$ & & $\begin{array}{l}\text { Burnout (SMBQ), work } \\
\text { ability I (WAI, adjusted), } \\
\text { stress (scale tools created for } \\
\text { this study), sleep (KSQ) }\end{array}$ & $\begin{array}{l}\text { T1: Just after first session } \\
\text { T2: Just after 12-week } \\
\text { program } \\
\text { T3: T2 }+6 \text { months } \\
\text { T4: T2 }+12 \text { months } \\
\end{array}$ & $\begin{array}{l}\text { 225-ha wild nature, incl. } \\
\text { forest, ponds, moorland, } \\
\text { hills; wooden house, } \\
\text { greenhouse }\end{array}$ & 3 \\
\hline $\begin{array}{l}\text { Nacau et al. } \\
\text { (2013) [67] } \\
\text { Japan }\end{array}$ & $\begin{array}{c}N=22 \\
\text { Age }=58.2 \\
\% \text { female }=81.8 \\
\% \text { Whites }=\text { N/A } \\
\text { Study group: Cancer, after treatment }\end{array}$ & $\begin{array}{l}12 \text { weeks, once per week, } 6 \text { hours. } 40 \\
\text { min. walks, } 60 \text { min. horticultural } \\
\text { therapy, } 90 \text { min. indoor yoga and } \\
\text { meditation ( } 1 \text { session), } 60 \text { min. } \\
\text { supportive group therapy ( } 5 \\
\text { sessions). } \\
\text { Homework: yoga (video). }\end{array}$ & & $\begin{array}{l}\text { Well-being (FACIT) incl. } \\
\text { physical, cancer fatigue } \\
\text { (CFS), quality of life (SF-36), } \\
\text { mood (POMS-SF), anxiety } \\
\text { (STAI), natural killer cell } \\
\text { activity (NK) }\end{array}$ & $\begin{array}{l}\text { T1: Just before treatment } \\
\text { T2: Just after } 12 \text { weeks' } \\
\text { treatment }\end{array}$ & $\begin{array}{l}\text { Park incl. forest, streams, } \\
\text { lawns, gardens; yoga and } \\
\text { meditation indoors in the } \\
\text { park }\end{array}$ & 3 \\
\hline $\begin{array}{l}\text { Combs et al. } \\
\text { (2016) [68] } \\
\text { USA }\end{array}$ & $\begin{array}{c}N=704(659) \\
\text { Age }=16 \\
\% \text { female }=32 \\
\% \text { Whites }=85 \\
\text { Study group: behavior/ substance/ } \\
\text { mood issues }\end{array}$ & $\begin{array}{l}\text { 90-day program. Nomadic hiking } \\
\text { and/or expeditions and tasks } \\
\text { associated with outdoor living. } \\
\text { Therapeutic tools: The wilderness } \\
\text { itself, CBT, choice therapy, family } \\
\text { systems, mindfulness techniques, } \\
\text { diet, physical exercise. Individual/ } \\
\text { group therapy sessions twice a week. }\end{array}$ & & $\begin{array}{l}\text { Psychological and } \\
\text { behavioral symptoms and } \\
\text { social functioning } \\
\text { (Y-OQ_SR) }\end{array}$ & $\begin{array}{l}\text { T1: At intake } \\
\text { T2: T1 }+3 \text { weeks } \\
\text { T3: } 11+5 \text { weeks } \\
\text { T4: At discharge } \\
\text { T5: T4 + } 6 \text { months } \\
\text { T6: T4 }+18 \text { months. }\end{array}$ & $\begin{array}{l}\text { Wilderness in undeveloped } \\
\text { areas }\end{array}$ & 3 \\
\hline $\begin{array}{l}\text { Russell } \\
\text { (2016) [69] } \\
\text { Canada }\end{array}$ & $\begin{array}{c}N=43(32) \\
\text { Age }=18-24(22.9) \\
\% \text { female }=0 \\
\% \text { Whites }=\text { N/A } \\
\text { Study group: } \\
\text { Substance abuse }\end{array}$ & $\begin{array}{l}\text { 90-day, 10-bed outdoor behavioral } \\
\text { healthcare program (Shunda Creek), } \\
\text { incl. weekly 1-5-day adventure trips } \\
\text { integrating mindfulness-based } \\
\text { experience (MBE) with } \\
\text { psychotherapy. }\end{array}$ & & $\begin{array}{l}\text { Subjective discomfort, } \\
\text { interpersonal relations, } \\
\text { social roles (OQ-45.2), } \\
\text { mindfulness (FFMQ) }\end{array}$ & $\begin{array}{c}\text { T1: At intake } \\
\text { T2: At discharge (average } \\
\text { T1 + } 93.7 \text { days) }\end{array}$ & $\begin{array}{l}\text { Wild nature, incl. } \\
\text { mountains }\end{array}$ & 3 \\
\hline $\begin{array}{l}\text { Russell et al. (2017) [70] } \\
\text { USA }\end{array}$ & $\begin{array}{c}N=168 \\
\text { Age }=21.5 \\
\% \text { female }=0 \\
\% \text { Whites }=40 \\
\text { Study group: Substance abuse }\end{array}$ & $\begin{array}{l}\text { 90-day outdoor behavioral healthcare } \\
\text { program (Shunda Creek): Weekly } \\
\text { 1-5-day adventure trips integrating } \\
\text { MBE with psychotherapy. }\end{array}$ & & $\begin{array}{c}\text { Helpfulness and } \\
\text { mindfulness (subscales of } \\
\text { OQ-45.2), adventure } \\
\text { therapy experience (ATES) }\end{array}$ & $\begin{array}{l}\text { T1: At admission } \\
\text { T2-T13: Every second week } \\
\text { until discharge }\end{array}$ & $\begin{array}{l}\text { Wild nature, incl. } \\
\text { mountains }\end{array}$ & 3 \\
\hline
\end{tabular}


Table 1. Cont.

\begin{tabular}{|c|c|c|c|c|c|c|c|}
\hline $\begin{array}{l}\text { Study } \\
\text { Author, Year, Country }\end{array}$ & $\begin{array}{c}\text { Population } \\
N=\text { Included } \\
\text { (Completed/Follow-up), } \\
\text { Age = Range (Mean), Gender, } \\
\text { Ethnicity, Target Group }\end{array}$ & $\begin{array}{c}\text { Intervention } \\
\text { Duration, Content }\end{array}$ & $\begin{array}{c}\text { Comparators Duration, } \\
\text { Content }\end{array}$ & $\begin{array}{c}\text { Outcomes } \\
\text { Measurements, Tools }\end{array}$ & $\begin{array}{c}\text { Time } \\
\text { Intervention, Timings of } \\
\text { Data Collection }\end{array}$ & $\begin{array}{c}\text { Setting } \\
\text { Location of Study Group }\end{array}$ & $\begin{array}{l}\text { EPHPP } \\
\text { Quality }\end{array}$ \\
\hline $\begin{array}{l}\text { Russell } \\
\text { (2018) [71] } \\
\text { Canada }\end{array}$ & $\begin{array}{c}N=57(46) \\
\text { Age }=12-17(16.6) \\
\% \text { female }=43.9 \\
\% \text { Whites }=57.9 \\
\text { Study group: 74\% diagnoses, } \\
\text { ADHD/substance use }\end{array}$ & $\begin{array}{c}\text { 8-week, 15-bed program: family } \\
\text { therapy, daily individual/group } \\
\text { therapy, educational programming. } \\
\text { Base camp model: Adventure } \\
\text { therapy and development of } \\
\text { mindfulness skills. }\end{array}$ & & $\begin{array}{l}\text { Emotional and behavioral } \\
\text { symptoms (Y-OQ SR 2.0), } \\
\text { mindfulness (CAMM) }\end{array}$ & $\begin{array}{l}\text { T1: Just before treatment } \\
\text { T2: Just after } 8 \text { weeks' } \\
\text { treatment }\end{array}$ & $\begin{array}{l}\text { Wild nature, incl. } \\
\text { mountains }\end{array}$ & 3 \\
\hline $\begin{array}{l}\text { Chapman et al. (2018) [72] } \\
\text { Canada }\end{array}$ & $\begin{array}{c}N=177 \\
\text { Age }=18-24(21.5) \\
\% \text { female }=0 \\
\% \text { Whites }=42.1 \\
\text { Study group: } \\
\text { Substance use }\end{array}$ & $\begin{array}{l}\text { 90-day outdoor behavioral healthcare } \\
\text { program (Shunda Creek): Weekly } \\
\text { 1-5-day adventure trips integrating } \\
\text { MBE with psychotherapy. }\end{array}$ & & $\begin{array}{l}\text { Subjective discomfort, } \\
\text { interpersonal relations, } \\
\text { social roles (OQ-45.2) }\end{array}$ & $\begin{array}{c}\text { T1: At intake } \\
\text { T2: At discharge (average } \\
\text { T1 + } 79.6 \text { days) }\end{array}$ & $\begin{array}{l}\text { Wild nature, incl. } \\
\text { mountains }\end{array}$ & 3 \\
\hline $\begin{array}{l}\text { Unsworth et al. } \\
\text { (2016) [73] } \\
\text { study 2 } \\
\text { USA }\end{array}$ & $\begin{array}{c}N=39 \\
\text { Age }=21 \\
\% \text { female }=64.1 \\
\% \text { Whites }=\text { N/A } \\
\text { Study group: No diagnosis }\end{array}$ & $\begin{array}{l}3 \text { days' Aztec adventure camp in } \\
\text { nature, incl. 15 min. . formal daily } \\
\text { morning meditation, and } \\
\text { encouragement to continue } \\
\text { mindfulness practice throughout the } \\
\text { day. }\end{array}$ & & $\begin{array}{l}\text { Self-nature } \\
\text { interconnectedness, } \\
\text { nature in self (INS), } \\
\text { mindfulness (FMI) }\end{array}$ & $\begin{array}{l}\text { T1: Just before treatment } \\
\text { T2: Just after } 3 \text { days' } \\
\text { treatment }\end{array}$ & Wild nature & 3 \\
\hline
\end{tabular}

Outcome measures: Beck Depression Inventory (BDI), Hamilton Rating Scale for Depression (HRSD), Montgomery-Åsberg Depression Rating Scale (MADRS), Depression Anxiety Stress
Scale (DASS21), Workers Stress Response Inventory (WSRI), State-Trait Anxiety Inventory (STAI), Perceived Stress Scale (PSS), Swedish Core Affect Scale (SCAS), Positive and Negative Affect Scale (PANAS), Psychological General Well-Being Index (PGWBI), Patient Reported Outcomes Measurement Information System (PROMIS), Profile of Mood States short form (POMS-SF), Rosenberg's Self-Esteem Scale (RSE), self-efficacy scale from Copenhagen Psychosocial Questionnaire (COPSOQ-II), Recovery Experience Questionnaire (REQ), Happiness Index for Koreans (HI-K), Elevation Experience Scale (EES), Sense of Meaning Scale (SMS), State Hope Scale (SHS), Gratitude Scale (GS), Self-Concordant Motivation (SCM), General Sense of Connectedness (GSC), Five-Facet Mindfulness Questionnaire (FFMQ), Child and Adolescent Mindfulness Measure (CAMM), Freiburg Mindfulness Inventory (FMI), State Mindfulness Scale (SMS), Cancer Fatigue Scale (CS), Shirom-Melamed Burnout Questionnaire (SMBQ), Malash Burnout Inventory-General Survey (MBI-GS), Visual Analog Scale (VAS), Brief Symptom Inventory (BSI), Functional Assessment of Chronic Illness Therapy-Spiritual Well-Being Scale (FACIT), Irvine's Spiritual Well-Being Scale (ISWBS), Transmutation Change Questionnaire (TCQ), Short Form-36 to measure health-related quality of life (SF-36), Quality of Life (QoL), EuroQol Visual Analog Scale (EQ-VAS), Basic Nordic Sleep Questionnaire (BNSQ), Karolinska Sleep Questionnaire (KSQ),Work Ability Index (WAI), Paffenbarger Physical Activity Questionnaire (PPAQ), Prosocial Orientation (PSO), Connectedness to Nature Scale (CNS), Engaging with Beauty Scale (EWB), Inclusion of Nature in Self (INS), Outcome Rating Scale (everyday functioning), Outcome Rating Scale Youth Outcome Questionnaire Self-Report (Y-OO SR), Outcome Questionnaire measuring psychological and behavioral symptoms (OQ-45.2), Adventure Therapy Experience (ATES), Letter-Digit Substitution Test (LDST), Trail-Making Test (TMT), heart rate (HR), heart rate variability (HRV), body mass index (BMI), lipid levels, lipid particle size, high sensitivity C-reactive protein, biomarkers IL-6 and IL-10, salivary cortisol level, pulse rate, systolic and diastolic blood pressure (BP), natural killer cell activity calcein-AM release assay using NK-sensitive K-562 cells as a target (NK). 


\subsection{Intervention Characteristics}

The interventions were highly heterogeneous concerning length, setup, and content, as well as the amount and type of mindfulness, and the choice of natural setting. All studies had psychological endpoints, and the most prevalent outcomes were psychological wellbeing or positive emotions $(\mathrm{k}=14)$, attention $(k=7)$, depression $(k=5)$, and anxiety $(k=4)$. Physiological endpoints were reported in eight studies, all including cardiovascular system outcomes, e.g., heart rate variability and blood pressure, and four studies reported outcomes related to the immune system, investigating e.g., natural killer cells and inflammation. Of studies including interpersonal outcomes $(k=7)$, four reported on interpersonal functioning and three on workability. The length of intervention varied from 15 minutes to 90 days, with follow-up data available for eight studies. The follow-up time ranged from two weeks to 18 months post-treatment.

Three main types of intervention emerged:

1. Short single-instruction intervention studies $(k=7)$ aimed at healthy participants, who were guided either to be mindful on their own while sitting or walking, or to be more extensively mindful in their everyday lives.

2. Weekly meetings (once or more per week) targeting stressed, anxious, or depressed people $(\mathrm{k}=6)$, mostly with gardening activities and psychotherapy. One study stood out in this format as only containing meditation training.

3. Residential interventions ( $k=11$ ), of which five were wilderness therapy of several weeks' duration. Participants in these studies were young people, mostly males diagnosed with substance use disorders. The other six residential interventions were shorter and in diverse settings.

Among the interventions reporting on formal meditation $(\mathrm{k}=8)$, three had full meditation training protocols: MBSR, MBCT [74], and restoration skill training (ReST) [54]. The MBSR and MBCT were integrated in extended nature-based interventions, with only ReST being a stand-alone program. Five studies mainly included concentration meditation, while open-awareness meditation was exclusively practiced in two programs. Among the informal mindfulness interventions $(k=8)$, four gave brief guidance toward the present moment, and three investigated a specific program of mindfulness-based experience [69] that integrated psychotherapy with mindfulness on adventure trips. The interventions' natural environments varied widely, from designed small gardens to vast wild nature areas. The amount of time devoted to guided mindfulness was less than five hours in all but two studies.

\subsection{Pooled Effect Sizes and Between-Study Differences}

The overall combined effect size from pre- to post-treatment across outcomes and designs was significant and of medium size $(g=0.54, p<0.001$; see Table 2). Studies employing an open, non-controlled design, again across outcomes, revealed a significant effect of medium size $(g=0.66$, $p<0.001)$; the same was true for studies with passive control groups $(g=0.58, p<0.001)$. Studies using active control groups also revealed a significant effect, albeit small in size $(g=0.26, p=0.023)$. Only one intervention was found that had a natural setting but without mindfulness as an active control group, and only one intervention included manual-based stand-alone mindfulness conducted in nature. The effects of these types of intervention were therefore not calculated. 
Table 2. Results from overall and subgroup meta-analyses.

\begin{tabular}{|c|c|c|c|c|c|c|c|c|c|c|c|}
\hline & \multicolumn{2}{|c|}{ Sample Size } & \multicolumn{4}{|c|}{ Heterogeneity } & \multicolumn{3}{|c|}{ Global Effect Aizes } & \multirow{2}{*}{ Fail-Safe $\mathrm{N}^{\mathrm{c}}$} & \multirow{2}{*}{ Criterior } \\
\hline & $\mathrm{k}$ & $N$ & $Q^{b}$ & $\mathrm{df}$ & $p$ & $\mathrm{I}^{2}$ & Hedges' $g$ & $95 \% \mathrm{CI}$ & $p$ & & \\
\hline Outcome from pre- to post-treatment & & & & & & & & & & & \\
\hline Combined effect across designs and outcomes & 25 & & 241.1 & 24 & $<0.001$ & 90.0 & 0.54 & $0.34-0.75$ & $<0.001$ & 2146 & 135 \\
\hline Adjusted for publication bias ${ }^{a}$ & 32 & & & & & & 0.83 & $0.55-0.91$ & & & \\
\hline Open trials & 13 & 1737 & 170.4 & 12 & $<0.001$ & 93.0 & 0.66 & $0.38-0.94$ & $<0.001$ & 1211 & 75 \\
\hline Adjusted for publication bias & 15 & & & & & & 0.76 & $0.50-1.02$ & & & \\
\hline Studies with passive control group & 6 & 821 & 10.3 & 5 & 0.068 & 51.4 & 0.58 & $0.34-0.82$ & $<0.001$ & 67 & 45 \\
\hline Studies with active control group & 7 & 900 & 12.1 & 7 & 0.096 & 42.3 & 0.26 & $0.04-0.49$ & 0.023 & 11 & 50 \\
\hline \multicolumn{12}{|l|}{ Combined effect across desions for each type of outcome } \\
\hline Psychological & 24 & 2990 & 215.7 & 23 & $<0.001$ & 89.3 & 0.55 & $0.36-0.74$ & $<0.001$ & 2169 & 130 \\
\hline Adjusted for publication bias & 30 & & & & & & 0.69 & $0.52-0.87$ & & & \\
\hline Adjusted for publication bias & 1 & & & & & & 0.29 & $0.02-0.55$ & & & \\
\hline Social & 4 & 432 & 3.9 & 2 & 0.143 & 48.6 & 0.39 & $0.13-0.65$ & 0.004 & 13 & 25 \\
\hline Adjusted for publication bias & 5 & & & & & & 0.22 & $-0.03-0.48$ & & & \\
\hline \multicolumn{12}{|l|}{ Outcome from pre-treatment to follow-up } \\
\hline Combined effect across designs & 8 & 1071 & 13.5 & 7 & 0.060 & 48.3 & 0.56 & $0.34-0.78$ & $<0.001$ & 97 & 50 \\
\hline Adjusted for publication bias & 11 & & & & & & 0.73 & $0.59-0.86$ & & & \\
\hline Open trials & 4 & 791 & 8.9 & 4 & 0.064 & 55.2 & 0.66 & $0.39-0.92$ & $<0.001$ & 66 & 35 \\
\hline \multirow{2}{*}{\multicolumn{12}{|c|}{ Categorical moderators }} \\
\hline \multicolumn{6}{|l|}{$\begin{array}{l}\text { Categorical moderators } \\
\text { Type of nature: }\end{array}$} & & & & & & \\
\hline Garden/park & 8 & 501 & 17.3 & 8 & 0.027 & 53.7 & 0.33 & $0.09-0.56$ & 0.008 & 28 & 55 \\
\hline Wild/forest & 15 & 1578 & 169.5 & 13 & $<0.001$ & 92.3 & 0.66 & $0.40-0.93$ & $<0.001$ & 14 & 80 \\
\hline Adjusted for publication bias & 17 & & & & & & 0.80 & $0.56-1.04$ & & & \\
\hline $\begin{array}{l}\text { Formal } \\
\text { Adjusted for publication bias }\end{array}$ & 10 & & & & & & 0.31 & $0.08-0.53$ & & & \\
\hline Informal & 8 & 1309 & 127.6 & 7 & $<0.001$ & 94.5 & 0.80 & $0.38-1.23$ & $<0.001$ & 463 & 50 \\
\hline Between-group difference & & & 3.1 & 1 & 0.078 & & & & & & \\
\hline Trait building & 4 & 276 & $\begin{array}{l}0.1 \\
14.8\end{array}$ & 3 & 0.002 & 79.8 & 10 & $-0.49-0.69$ & 0.732 & & \\
\hline State inducing & 21 & 2624 & 214.6 & 20 & $<0.001$ & 90.7 & 0.62 & $0.41-0.83$ & $<0.001$ & 2041 & 115 \\
\hline Between-group difference & & & $\begin{array}{c}2.60 \\
2.6\end{array}$ & 1 & 0.107 & & & & & & \\
\hline
\end{tabular}

${ }^{a}$ The possibility of publication bias was examined with funnel plots and Egger's tests followed by imputation of missing studies. $(\mathrm{k})=\mathrm{k}+$ number of imputed studies. ${ }^{\mathrm{b}}$ For the Q-statistic, $p$-values of $<0.05$ are considered indicative of heterogeneity. ${ }^{c}$ The fail-safe $N$ was calculated for statistically significant findings to examine the robustness of these findings, representing the number of non-significant studies that would bring the $p$-value to non-significance (i.e., $p>0.05$ ). 
When the effects from pre- to post-treatment were evaluated for individual outcomes across designs, the effect on psychological outcomes was significant and of medium size $(g=0.55, p<0.001)$. The effects on social $(g=0.39, p=0.004)$ and physical $(g=0.36, p=0.011)$ outcomes were significant and of small size.

Concerning effects at follow-up, the combined effect across designs and outcomes was significant and of moderate size $(g=0.56, p<0.001)$. Only the studies employing an open trial design were sufficient in number to perform a separate meta-analysis, which also revealed a significant effect of medium size $(g=0.66, p<0.001)$.

A number of planned moderation analyses were conducted, none of which were statistically significant. However, type of nature $(p=0.068, Q=3.4)$ and trended toward significance. Interventions in wild/forest environments obtained a numerically larger effect $(g=0.66)$ than interventions in garden/park environments $(g=0.33)$. Moreover, the type of mindfulness $(p=0.078, Q=0.31)$ did as well trend towards significance, and informal mindfulness interventions obtained a numerically larger effect $(g=0.80)$ than formal mindfulness interventions $(g=0.37)$. The effect size for inducing state mindfulness was larger $(g=0.62)$ than for building trait mindfulness $(g=0.10)$. No significance was found for this moderator $(p=0.107)$. Only two interventions primarily used open awareness, and the moderating effect of this type of mindfulness was therefore not calculated (see Table 3).

Table 3. Results from meta-regression-based moderation analyses.

\begin{tabular}{cccc}
\hline Moderator & B & SE & $p$ \\
\hline Participant characteristics & & & \\
Mean sample age & $<0.01$ & 0.01 & 0.893 \\
\% women & $<-0.01$ & $<0.01$ & 0.425 \\
\% Whites & 0.01 & 0.01 & 0.506 \\
\hline Intervention characteristics & & & \\
Intervention duration & $<0.01$ & $<0.01$ & 0.716 \\
$\quad$ Sessions with & $<-0.01$ & 0.01 & 0.597 \\
mindfulness & & &
\end{tabular}

Note: $\mathrm{B}=$ Unstandardized beta coefficient; $\mathrm{SE}=$ Standard error of $\mathrm{B} ; p=$ level of significance.

\subsection{Publication Bias}

For all statistically significant results, publication bias was evaluated. Eight analyses were adjusted for possible publication bias, which in only one case (i.e., effect on social outcomes) changed the result from significance to non-significance. Seven out of 13 studies failed to meet the criterion for the fail-safe $N$, indicating lack of robustness for approximately half of the results.

\section{Discussion}

We were able to identify 25 independent studies that met our criteria. Across the designs of these studies, an initial synthesis showed overall positive effects of mindfulness in natural settings evaluated in both open trials and controlled trials using non-active control groups. These results support our hypothesis that context may play a significant role in the benefits of mindfulness-based interventions. This may be explained by the experience of the natural environment, which is so fascinating that it calls for soft attention, thereby allowing disengagement [4]. This is comparable to "letting go" in mindfulness, where the meditator is guided not to mentally hold on to anything, and not to push anything away [6]. Another explanation could be that natural stimuli occupy the attention [4] and consequently reduce the tendency for the mind to wander [5], which is another aim in mindfulness training. While experienced meditators may be able to stay present during meditation, exposure to nature may support inexperienced or otherwise challenged meditators who would otherwise be at risk of losing concentration completely or becoming emotionally overwhelmed [4]. 
A number of moderation analyses were conducted. Forests/wild nature and informal mindfulness were found to trend-wise increase positive health outcomes based on large differences in effect size, although these were not significant. The lack of significance may be due to the low number of studies, and should be interpreted according to the effect sizes. The numerically larger positive effect of natural settings characterized as wild supports the findings by Grahn and Stigsdotter [75], suggesting that stressed individuals prefer natural environments that are wild and untouched and offer a variety of species but are still experienced as safe.

Informal mindfulness tended to moderate positive health outcomes compared with formal mindfulness, as did inducing state mindfulness compared with building trait-mindfulness. Both may be explained by the outward focus, which may be more beneficial in a natural setting, as it allows more contact with nature. Furthermore, the possibility of engaging in activities during informal mindfulness may explain the more positive outcomes. Corazon, Schilhab, and Stigsdotter [76] argue that bodily involvement with the environment is important in nature-based therapy, as it strengthens the memory of experiences in nature, and thereby prolongs and confirms the therapeutic effect.

\subsection{Implications for Research and Practice}

The field of nature-based mindfulness is in its infancy, and is not yet defined; our study only suggests some structure. One of the aspects that are still in need of investigation is whether certain types of mindfulness are more suited than others for training and use in natural settings, and whether this depends on the characteristics of the natural setting and other components of the intervention. Informal mindfulness is compatible with ordinary activities [5], such as walking a forest trail e.g., [62], and has been shown to be a tool for healthy people to enhance the positive effects of contact with nature $[63,77]$. Forest bathing is a research field that addresses this [25], but a systematic approach to mindfulness is still needed in this context. In nature-based therapy, on the other hand, informal mindfulness enhances awareness of negative thought patterns, which seem easier to detect in natural settings, and this is of value in a therapeutic context $[66,78,79]$.

Lymeus et al. [54] argue that the practice of open-monitoring meditation (comparable to open-awareness meditation) in natural environments is superior to concentration meditation, as it allows natural stimuli softly and effortlessly to hold the attention to the present moment. Due to the scarcity of available studies, it was unfortunately not possible to compare open-awareness meditation with concentration meditation as moderators of positive health outcomes in this review. It is recommended that future studies should address this gap in knowledge, and should also carefully define and describe the way the mindfulness is conducted and the characteristics of the natural setting in which the therapy takes place. In addition, it seems reasonable to not only include nature in health promoting activities [23], but also to include informal mindfulness (i.e., guided attention to the senses with an attitude of non-judgment and openness) in nature-based therapy. Formal meditation in natural settings also seems to be a promising tool, and further research is needed to provide guidelines for such practice.

\subsection{Limitations}

The rather low quality of the included studies poses a threat to the validity of the findings, which need to be confirmed in high-quality studies. In particular, blinding and selection bias were issues, and only a few trials could be categorized as clinical trials according to the EPHPP assessment criteria. With only 25 studies included in this review, and in light of the heterogeneity of the participants and intervention characteristics, the generalizability is limited. Furthermore, a different definition of mindfulness than that employed in this study might affect the character and number of studies included. The included studies would preferably define mindfulness as containing an attitude of e.g., warmth and non-judgment, but meditation practices are rarely described in detail, and such narrow inclusion criteria would at present exclude most studies. 


\section{Conclusions}

This systematic review and meta-analysis shows that nature-based mindfulness has had a positive effect on psychological, physical, and social conditions. Furthermore, nature-based mindfulness is moderately superior to mindfulness conducted in non-natural settings. However, at this point we know very little about the effect of different types of mindfulness, and more research is needed to understand what an optimal mindfulness intervention in a nature-based setting should consist of. Mindfulness in wild nature seems to be more beneficial than mindfulness in more cultivated settings, but the importance of the setting needs further investigation.

Author Contributions: Conceptualization, D.D., U.S., J.D. and M.O.; Data curation, D.D.; Formal analysis, D.D. and M.O.; Investigation, D.D., I.L. and D.P.; Methodology, D.D. and M.O.; Writing—original draft, D.D. and M.O.; Writing-review \& editing, D.D., I.L., D.P., U.S., J.D. and M.O.

Funding: This research received no external funding.

Acknowledgments: We would like to give thanks to Sus Sola Corazon and Mimi Y. Mehlsen for inspiration and advice.

Conflicts of Interest: The authors declare no conflict of interest.

\section{References}

1. Lymeus, F.; Lundgren, T.; Hartig, T. Attentional effort of beginning mindfulness training is offset with practice directed toward images of natural scenery. Environ. Behav. 2017, 49, 536-559. [CrossRef]

2. Ambrose-Oji, B. Mindfulness Practice in Woods and Forests: An Evidence Review. In Research Report for The Mersey Forest, Forest Research; Alice Holt Lodge Farnham: Surrey, UK, 2013.

3. Schutte, N.S.; Malouff, J.M. Mindfulness and connectedness to nature: A meta-analytic investigation. Pers. Individ. Differ. 2018, 127, 10-14. [CrossRef]

4. Kaplan, S. Meditation, Restoration, and the Management of Mental Fatigue. Environ. Behav. 2001, 33, 480-506. [CrossRef]

5. Bishop, S.; Lau, M.; Shapiro, S.; Carlson, L.; Anderson, N.; Carmody, J.; Devins, G. Mindfulness: A Proposed Operational Definition. Clin. Psychol. Sci. Pract. 2004, 11, 230-241. [CrossRef]

6. Kabat-Zinn, J. Full Catastrophe Living: Using the Wisdom of Your Body and Mind to Face Stress, Pain, and Illness; rev. ed.; Bantam Books: New York, NY, USA, 2013.

7. Dahl, C.J.; Lutz, A.; Davidson, R.J. Reconstructing and deconstructing the self: Cognitive mechanisms in meditation practice. Trends Cogn. Sci. 2015, 19, 515-523. [CrossRef] [PubMed]

8. Johnson, S.; Gur, R.M.; David, Z.; Currier, E. One-session mindfulness meditation: A randomized controlled study of effects on cognition and mood. Mindfulness 2015, 6, 88-98. [CrossRef]

9. Piet, J.; Hougaard, E. The effect of mindfulness-based cognitive therapy for prevention of relapse in recurrent major depressive disorder: A systematic review and meta-analysis. Clin. Psychol. Rev. 2011, 31, 1032-1040. [CrossRef]

10. Sedlmeier, P.; Eberth, J.; Schwarz, M.; Zimmermann, D.; Haarig, F.; Jaeger, S.; Kunze, S. The psychological effects of meditation: A meta-analysis. Psychol. Bull. 2012, 138, 1139-1171. [CrossRef]

11. Chiesa, A.; Serretti, A. Mindfulness-Based Interventions for Chronic Pain: A Systematic Review of the Evidence. J. Altern. Complement. Med. 2011, 17, 83-93. [CrossRef]

12. Black, D.S.; Slavich, G.M. Mindfulness meditation and the immune system: A systematic review of randomized controlled trials. Ann. N. Y. Acad. Sci. 2016, 1373, 13-24. [CrossRef]

13. Beauchemin, J.; Hutchins, T.L.; Patterson, F. Mindfulness meditation may lessen anxiety, promote social skills, and improve academic performance among adolescents with learning disabilities. Complement. Health Pract. Rev. 2008, 13, 34-45. [CrossRef]

14. De Vibe, M. Mindfulness based stress reduction (MBSR) for improving health, quality of life, and social functioning in adults. Campbell Syst. Rev. 2012, 8, 1-127. [CrossRef]

15. Gu, J.; Strauss, C.; Bond, R.; Cavanagh, K. How do mindfulness-based cognitive therapy and mindfulness-based stress reduction improve mental health and wellbeing? A systematic review and meta-analysis of mediation studies. Clin. Psychol. Rev. 2015, 37, 1-12. [CrossRef] [PubMed] 
16. Bratman, G.N.; Hamilton, J.P.; Daily, G.C. The impacts of nature experience on human cognitive function and mental health. Ann. N. Y. Acad. Sci. 2012, 1249, 118-136. [CrossRef] [PubMed]

17. Mausner, C. A Kaleidoscope model: Defining natural environments. J. Environ. Psychol. 1996, 16, 335-348.

18. Hartig, T.; Mitchell, R.; De Vries, S.; Frumkin, H. Nature and Health. Annu. Rev. Public Health 2014, 35, 207-228. [CrossRef] [PubMed]

19. Mcmahan, E.A.; Estes, D. The effect of contact with natural environments on positive and negative affect: A meta-analysis. J. Posit. Psychol. 2015, 10, 507-519. [CrossRef]

20. Beyer, K.M.M.; Kaltenbach, A.; Szabo, A.; Bogar, S.; Nieto, F.J.; Malecki, K.M. Exposure to neighborhood green space and mental health: Evidence from the survey of the health of Wisconsin. Int. J. Environ. Res. Public Health 2014, 11, 3453-3472. [CrossRef]

21. Kuo, M. How might contact with nature promote human health? Promising mechanisms and a possible central pathway. Front. Psychol. 2015, 6, 1093. [CrossRef]

22. De Vries, S.; Van Dillen, S.M.; Groenewegen, P.P.; Spreeuwenberg, P. Streetscape greenery and health: Stress, social cohesion and physical activity as mediators. Soc. Sci. Med. 2013, 94, 26-33. [CrossRef]

23. Frumkin, H.; Bratman, G.N.; Breslow, S.J.; Cochran, B.; Kahn, P.H., Jr.; Lawler, J.J.; Levin, P.S.; Tandon, P.S.; Varanasi, U.; Wolf, K.L.; et al. Nature Contact and Human Health: A Research Agenda. Environ. Health Perspect. 2017, 125, 075001. [CrossRef] [PubMed]

24. Li, Q.; Kawada, T. Effect of forest environments on human natural killer (NK) activity. Int. J. Immunopathol. Pharmacol. 2011, 24, 39S-44S. [PubMed]

25. Hansen, M.M.; Jones, R.; Tocchini, K. Shinrin-Yoku (Forest Bathing) and Nature Therapy: A State-of-the-Art Review. Int. J. Environ. Res. Public Health 2017, 14, 851. [CrossRef] [PubMed]

26. Oh, B.; Lee, K.J.; Zaslawski, C.; Yeung, A.; Rosenthal, D.; Larkey, L.; Back, M. Health and well-being benefits of spending time in forests: Systematic review. Environ. Health Prev. Med. 2017, 22, 71. [CrossRef] [PubMed]

27. Bettmann, J.E.; Gillis, H.L.; Speelman, E.A.; Parry, K.J.; Case, J.M. A Meta-analysis of Wilderness Therapy Outcomes for Private Pay Clients. J. Child Fam. Stud. 2016, 25, 2659-2673. [CrossRef]

28. Bowen, D.J.; Neill, J.T.; Crisp, S.J. Wilderness adventure therapy effects on the mental health of youth participants. Eval. Program Plan. 2016, 58, 49-59. [CrossRef]

29. Soga, M.; Gaston, K.J.; Yamaura, Y. Gardening is beneficial for health: A meta-analysis. Prev. Med. Rep. 2017, 5, 92-99. [CrossRef]

30. Shapiro, S.L.; Carlson, L.E.; Astin, J.A.; Freedmanm, B. Mechanisms of mindfulness. J. Clin. Psychol. 2006, 62, 373-386. [CrossRef]

31. Li, D.; Sullivan, W.C. Impact of views to school landscapes on recovery from stress and mental fatigue. Landsc. Urban Plan. 2016, 148, 149-158. [CrossRef]

32. Evensen, K.H.; Raanaas, R.K.; Hagerhall, C.M.; Johansson, M.; Patil, G.G. Restorative elements at the computer workstation: A comparison of live plants and inanimate objects with and without window view. Environ. Behav. 2015, 47, 288-303. [CrossRef]

33. Stevenson, M.P.; Schilhab, T.; Bentsen, P. Attention Restoration Theory II: A systematic review to clarify attention processes affected by exposure to natural environments. J. Toxicol. Environ. Health Part B 2018, 21, 227-268.

34. Kaplan, S.; Kaplan, R. The Experience of Nature: A Psychological Perspective; Cambridge University Press: New York, NY, USA, 1989.

35. Kaplan, S. The restorative benefits of nature: Towards an integrative framework. J. Environ. Health 1995, 16, 169-182. [CrossRef]

36. Jiang, B.; Schmillen, R.; Sullivan, W.C. How to waste a break: Using portable electronic devices substantially counteracts attention enhancement effects of green spaces. Environ. Behav. 2018. [CrossRef]

37. Moher, D.; Shamseer, L.; Clarke, M.; Ghersi, D.; Liberati, A.; Petticrew, M.; Shekelle, P.; A Stewart, L. Preferred reporting items for systematic review and meta-analysis protocols (PRISMA-P) 2015 statement. Syst. Rev. 2015, 4, 1. [PubMed]

38. Mennin, D.S.; Ellard, K.K.; Fresco, D.M.; Gross, J.J. United We Stand: Emphasizing Commonalities Across Cognitive-Behavioral Therapies. Behav. Ther. 2013, 44, 234-248.

39. Mennin, D.S.; Fresco, D.M. Emotion regulation therapy. In Handbook of Emotion Regulation, 2nd ed.; Guilford Press: New York, NY, USA, 2014; pp. 469-490. 
40. Thomas, B.H.; Ciliska, D.; Dobbins, M.; Micucci, S. A process for systematically reviewing the literature: Providing the research evidence for public health nursing interventions. Worldviews Evid. Based Nurs. 2004, 1, 176-184. [CrossRef] [PubMed]

41. Hedges, L.V.; Olkin, I. Statistical Methods for Meta-Analysis; Academic Press: London, UK, 1985.

42. Cohen, J. Applied Power Analysis for the Behavioral Science; Lawrence Erlbaum: Hillsdale, NJ, USA, 1988.

43. Hedges, L.V.; Pigott, T.D. The power of statistical tests in meta-analysis. Psychol. Methods 2001, 6, $203-217$.

44. Higgins, J.P.T.; Thompson, S.G. Quantifying heterogeneity in a meta-analysis. Stat. Med. 2002, 21, 1539-1558. [CrossRef]

45. Egger, M.; Smith, G.D.; Schneider, M.; Minder, C. Bias in meta-analysis detected by a simple, graphical test. BMJ 1997, 315, 629-634.

46. Duval, S.; Tweedie, R. Trim and Fill: A Simple Funnel-Plot-Based Method of Testing and Adjusting for Publication Bias in Meta-Analysis. Biometrics 2000, 56, 455-463. [CrossRef]

47. Rosenthal, R. The file drawer problem and tolerance for null results. Psychol. Bull. 1979, 86, 638-641. [CrossRef]

48. Ballew, M.T.; Omoto, A.M. Absorption: How Nature Experiences Promote Awe and Other Positive Emotions. Ecopsychology 2018, 10, 26-35.

49. Shin, Y.; Kim, D.; Jung-Choi, K.; Son, Y.; Koo, J.; Min, J.; Chae, J. Differences of psychological effects between meditative and athletic walking in a forest and gymnasium. Scand. J. For. Res. 2012, 28, 1-9.

50. Passmore, H.; Holder, M. Noticing nature: Individual and social benefits of a two-week intervention. J. Posit. Psychol. 2017, 12, 537-546.

51. Kim, W.; Lim, S.; Chung, E.; Woo, J. The Effect of Cognitive Behavior Therapy-Based Psychotherapy Applied in a Forest Environment an Physiological Changes and Remission of Major Depressive Disorder. Psychiatry Investig. 2009, 6, 245-254. [PubMed]

52. Willert, M.V.; Wieclaw, J.; Thulstrup, A.M. Rehabilitation of individuals on long-term sick leave due to sustained stress-related symptoms: A comparative follow-up study. Scand. J. Public Health 2014, 42, 719-727. [CrossRef] [PubMed]

53. Vujcic, M.; Tomicevic-Dubljevic, J.; Grbic, M.; Lecic-Tosevski, D.; Vukovic, O.; Toskovic, O. Nature based solution for improving mental health and well-being in urban areas. Environ. Res. 2017, 158, 385-392. [PubMed]

54. Lymeus, F.; Lindberg, P.; Hartig, T. Building mindfulness bottom-up: Meditation in natural settings supports open monitoring and attention restoration. Conscious. Cogn. 2018, 59, 40-56. [PubMed]

55. Han, J.-W.; Choi, H.; Jeon, Y.-H.; Yoon, C.-H.; Woo, J.-M.; Kim, W. The Effects of Forest Therapy on Coping with Chronic Widespread Pain: Physiological and Psychological Differences between Participants in a Forest Therapy Program and a Control Group. Int. J. Environ. Res. Public Health 2016, 13, 255.

56. Won, S.; Chang, S.; Poung, Y. The influence of forest therapy camp on depression in alcoholics. Environ. Health Prev. Med. 2012, 17, 73-76.

57. Warber, S.L.; Ingerman, S.; Moura, V.L.; Wunder, J.; Northrop, A.; Gillespie, B.W.; Durda, K.; Smith, K.; Rhodes, K.S.; Rubenfire, M. Healing the Heart: A Randomized Pilot Study of a Spiritual Retreat for Depression in Acute Coronary Syndrome Patients. Explore 2011, 7, 222-233. [CrossRef] [PubMed]

58. Sung, J.; Woo, J.; Kim, W.; Lim, S.; Chung, E. The Effect of Cognitive Behavior Therapy-Based "Forest Therapy" Program on Blood Pressure, Salivary Cortisol Level, and Quality of Life in Elderly Hypertensive Patients. Clin. Exp. Hypertens. 2012, 34,1-7. [PubMed]

59. Passmore, H.; Howell, A. Nature Involvement Increases Hedonic and Eudaimonic Well-Being: A Two-Week Experimental Study. Ecopsychology 2014, 6, 148-154.

60. Jung, W.H.; Woo, J.-M.; Ryu, J.S. Effect of a forest therapy program and the forest environment on female workers' stress. Urban For. Urban Green. 2015, 14, 274-281.

61. Yu, C.-P.; Lin, C.-M.; Tsai, M.-J.; Tsai, Y.-C.; Chen, C.-Y. Effects of Short Forest Bathing Program on Autonomic Nervous System Activity and Mood States in Middle-Aged and Elderly Individuals. Int. J. Environ. Res. Public Health 2017, 14, 897. [CrossRef] [PubMed]

62. Korpela, K.; Savonen, E.-M.; Anttila, S.; Pasanen, T.; Ratcliffe, E. Enhancing wellbeing with psychological tasks along forest trails. Urban For. Urban Green. 2017, 26, 25-30. [CrossRef]

63. Yang, C.-H.; Conroy, D.E. Feasibility of an Outdoor Mindful Walking Program for Reducing Negative Affect in Older Adults. J. Aging Phys. Act. 2019, 27, 18-27. [CrossRef] [PubMed] 
64. Corazon, S.S.; Nyed, P.K.; Sidenius, U.; Poulsen, D.V.; Stigsdotter, U.K. A Long-Term Follow-Up of the Efficacy of Nature-Based Therapy for Adults Suffering from Stress-Related Illnesses on Levels of Healthcare Consumption and Sick-Leave Absence: A Randomized Controlled Trial. Int. J. Environ. Res. Public Health 2018, 15, 137. [CrossRef] [PubMed]

65. Stigsdotter, U.K.; Corazon, S.S.; Sidenius, U.; Nyed, P.K.; Larsen, H.B.; Fjorback, L.O. Efficacy of nature-based therapy for individuals with stress-related illnesses: Randomised controlled trial. Br. J. Psychiatry 2018, 213, 404-411.

66. Sahlin, E.; Ahlborg, G.; Matuszczyk, J.V.; Grahn, P. Nature-Based Stress Management Course for Individuals at Risk of Adverse Health Effects from Work-Related Stress-Effects on Stress Related Symptoms, Workability and Sick Leave. Int. J. Environ. Res. Public Health 2014, 11, 6586-6611.

67. Nakau, M.; Imanishi, J.; Imanishi, J.; Watanabe, S.; Imanishi, A.; Baba, T.; Hirai, K.; Ito, T.; Chiba, W.; Morimoto, Y. Spiritual Care of Cancer Patients by Integrated Medicine in Urban Green Space: A Pilot Study. Explore 2013, 9, 87-90. [PubMed]

68. Combs, K.M.; Hoag, M.J.; Javorski, S.; Roberts, S.D. Adolescent Self-Assessment of an Outdoor Behavioral Health Program: Longitudinal Outcomes and Trajectories of Change. J. Child Fam. Stud. 2016, 25, 3322-3330.

69. Russell, K.C.; Gillis, H.L.L.; Heppner, W. An examination of mindfulness-based experiences through adventure in substance use disorder treatment for young adult males: A pilot study. Mindfulness 2016, 7, 320-328. [CrossRef]

70. Russell, K.C.; (Lee) Gillis, H.L.; Kivlighan, D.M. Process factors explaining psycho-social outcomes in adventure therapy. Psychotherapy 2017, 54, 273-280. [CrossRef] [PubMed]

71. Russell, K.C.; Gillis, H.L.; Law, L.; Couillard, J. A Pilot Study Examining Outcomes Associated with the Implementation of Progress Monitoring at a Substance Use Disorder Treatment Program for Adolescents. Child Youth Care Forum 2018, 47, 403-419.

72. Chapman, J.; Groark, S.; Beale, M.M.; Mandas, P.; Argo, K.; Gillis, H.L.L. The Relationship Between Self-Reported Prior Drug Use and Treatment Effectiveness in Substance Use Disorder during Outdoor Behavioral Healthcare Treatment for Young Adult Males. J. Ther. Sch. Programs 2018, 10, 3227.

73. Unsworth, S.; Palicki, S.-K.; Lustig, J. The Impact of Mindful Meditation in Nature on Self-Nature Interconnectedness. Mindfulness 2016, 7, 1052-1060.

74. Segal, Z.V.; Williams, J.M.G.; Teasdale, J.D. Mindfulness-Based Cognitive Therapy for Depression: A New Approach to Preventing Relapse; Guilford Press: New York, NY, USA, 2002.

75. Grahn, P.; Stigsdotter, U.K. The relation between perceived sensory dimensions of urban green space and stress restoration. Landsc. Urban Plan. 2010, 94, 264-275. [CrossRef]

76. Corazon, S.S.; Schilhab, T.S.S.; Stigsdotter, U.K. Developing the therapeutic potential of embodied cognition and metaphors in nature-based therapy: Lessons from theory to practice. J. Adventure Educ. Outdoor Learn. 2011, 11, 161-171.

77. Schuling, R.; Van Herpen, N.; De Nooij, R.; De Groot, W.T.; Speckens, A. Silent into Nature: Factors Enabling Improvement in a Mindful Walking Retreat in Nature of People with Psychological Symptoms. Ecopsychology 2018, 10, 77-86.

78. Poulsen, D.V.; Stigsdotter, U.K.; Djernis, D.; Sidenius, U. 'Everything just seems much more right in nature': How veterans with post-traumatic stress disorder experience nature-based activities in a forest therapy garden. Health Psychol. Open 2016, 3. [CrossRef] [PubMed]

79. Sidenius, U.; Stigsdotter, U.K.; Poulsen, D.V.; Bondas, T. “I look at my own forest and fields in a different way": The lived experience of nature-based therapy in a therapy garden when suffering from stress-related illness. Int. J. Qual. Stud. Health Well-Being 2017, 12, 1324700. [PubMed]

(C) 2019 by the authors. Licensee MDPI, Basel, Switzerland. This article is an open access article distributed under the terms and conditions of the Creative Commons Attribution (CC BY) license (http://creativecommons.org/licenses/by/4.0/). 